\title{
Pointes, hachoirs et marteaux
}

Variations sur les représentations de la violence des femmes au tournant $\mathrm{du} \mathrm{XX}^{\mathrm{e}}$ siècle

Spikes, cleavers and hammers. Variations on the representations of violence by women during the 20th century

\section{Eva Belgherbi}

\section{OpenEdition}

\section{Journals}

Édition électronique

URL : http://journals.openedition.org/cel/9572

DOI : $10.4000 /$ cel. 9572

ISSN : 2262-208X

Éditeur

École du Louvre

Référence électronique

Eva Belgherbi, «Pointes, hachoirs et marteaux », Les Cahiers de l'École du Louvre [En ligne], 15 | 2020,

mis en ligne le 03 novembre 2020, consulté le 21 décembre 2020. URL : http://

journals.openedition.org/cel/9572; DOI : https://doi.org/10.4000/cel.9572

Ce document a été généré automatiquement le 21 décembre 2020.

\section{cc) $(9)$}

Les Cahiers de l'École du Louvre sont mis à disposition selon les termes de la licence Creative Commons Attribution - Pas d'Utilisation Commerciale - Pas de Modification 4.0 International. 


\title{
Pointes, hachoirs et marteaux
}

\author{
Variations sur les représentations de la violence des femmes au tournant \\ du XX ${ }^{\mathrm{e}}$ siècle \\ Spikes, cleavers and hammers. Variations on the representations of violence by \\ women during the 20th century
}

\section{Eva Belgherbi}

"Armée d'un ciseau et d'un court marteau ${ }^{1}$ » : c'est ainsi qu'un journaliste décrit Camille Claudel (1864-1943) lors d'une visite dans son atelier du quai de Bourbon en 1905, mettant ainsi en exergue les outils de travail de la sculptrice prête à attaquer la matière. Dans la suite de l'entretien, Claudel revendique un travail difficile, ambitieux et solitaire ${ }^{2}$, se passant de main d'œuvre supplémentaire ${ }^{3}$. À travers cette présentation, la sculptrice se « virilise » en soulignant qu'elle s'attèle à une tâche jugée physiquement éprouvante qui outrepasse les habituelles assignations du genre féminin ${ }^{4}$. À la fin du XIX ${ }^{e}$ siècle, l'image de l'artiste taillant la pierre persiste au profit du sculpteur modeleur, alors même que la sculpture est une véritable entreprise collective mobilisant une vaste chaîne de métiers, de la mise au point du bloc de marbre à la fonte du bronze. L'un des gestes emblématiques de la sculpture demeure dans l'imaginaire collectif celui de la taille, comme le démontre l'historien de l'art Jean-François Corpataux dans son ouvrage Le Corps à l'œuvre ${ }^{5}$. En même temps, dès les années 1890, des recherches plastiques conduisent les artistes à redéfinir leur rapport à la matière, au bois ou à la pierre : certains entreprennent la taille directe sans passer par le modelage préalable de la forme, et entretiennent un rapport plus personnel à l'acte de sculpter, donnant une importance nouvelle à leurs outils ${ }^{6}$. Par un procédé métonymique, la pointe et le maillet restent dans l'imaginaire commun les attributs de la Sculpture; dans les représentations de l'artiste en sculpteur, les outils font partie de l'image, qu'ils soient posés aux pieds d'épigones de Pygmalion ou brandis dans le geste michelangelesque de l'attaque de la matière pour en dégager l'œuvre ${ }^{7}$. Au tournant du $\mathrm{XIX}^{\mathrm{e}}$ siècle, réinvestissant ces iconographies, des sculptrices posent dans des photographies reproduites dans les revues illustrées, se montrant en train de sculpter, posant à côté de leurs œuvres, et témoignant de leur pratique artistique, alors considérée comme un domaine plus masculin que la peinture, et inappropriée pour les 
femmes ${ }^{8}$. Cette conception de la sculpture comme art viril fait partie des arguments qui ont participé à retarder l'entrée des femmes artistes à l'École des beaux-arts, qui leur ouvre ses portes officiellement et partiellement en 1897, après des années de combats menés par la sculptrice Hélène Bertaux (1825-1909) et les membres de l'Union des Femmes Peintres et Sculpteurs qu'elle crée en $1881^{9}$.

2 La question de l'enseignement de domaines réservés aux hommes est aussi au cœur des revendications féministes en Europe à la fin des années 1890 : des groupes de femmes s'organisent, et ont parfois recours à la violence, à l'instar des suffragettes du Women's Social and Political Union (WSPU). En Grande-Bretagne, les membres de cette organisation fondée en 1903 à Manchester et menée par Emmeline Pankhurst (1858-1928) défilent dans les rues pour demander le droit de vote et affrontent la police, allant jusqu'à rendre les coups et dégrader des propriétés publiques, dont les œuvres d'art des musées. Le 10 mars 1914, la suffragette Mary Richardson (1889-1961) brise le verre de protection, lacère puis crève la toile de la Toilette de Vénus (dite Vénus Rokeby) de Diego Vélázquez, conservée à la National Gallery de Londres. Cet évènement destructeur a été largement analysé ${ }^{10}$, moins sa motivation et sa justification dans un mouvement plus global de révolte des femmes dans le domaine politique ainsi qu'artistique avec la redéfinition de la place des femmes comme véritables créatrices. Ces phénomènes sociaux s'accompagnent en effet du réinvestissement de modèles iconographiques déjà existants, produisant de nouvelles images de femmes s'introduisant dans les domaines masculins, politique et artistique, et porteuses d'une violence symbolique et parfois physique.

Cette contribution propose d'analyser la rhétorique des images créées dans un contexte de tensions entre la redéfinition de ce que peuvent les femmes en art ou en politique et une identité masculine qui serait menacée dans ses propres territoires. À travers quelques exemples de ces conquêtes politiques, institutionnelles ou symboliques dans les années 1890-1910 au cours desquelles les femmes s'imposent collectivement ou individuellement, par la force physique ou la voie légale, dans les domaines identifiés comme masculins que sont la politique et la sculpture. Une étude des photographies et caricatures révèle des disparités de genre plus vastes portées par des discours dont la violence est parfois de nature misogyne et souvent discriminante. Cette lutte de territoire produit des images, plus ou moins offensives, et fait l'objet de nombreuses caricatures, symptômes d'un contexte antiféministe plus large présent alors dans les milieux politiques et sociaux avant la Première Guerre mondiale, afin d'amoindrir les revendications des femmes - dont des sculptrices - et atténuer la menace qu'elles représentent pour les hommes sur fond de "guerre des sexes" réactivant les métaphores guerrières dont la « trouée féministe ${ }^{11}$ ».

\section{Versus Vénus : le corps des femmes à l'épreuve des altérations physiques et des combats politiques}

4 L'agression de la Toilette de Vénus (dite Vénus Rokeby), a retenu l'attention alors que de nombreux actes de vandalismes dans les musées ont déjà eu lieu avant 1914 sur des œuvres qui ne figuraient pas systématiquement des corps féminins ${ }^{12}$. L'attaque au hachoir ${ }^{13}$ de la Vénus par Mary Richardson s'inscrit dans une série de démonstrations de force dirigées contre la police et les pouvoirs publics, mais elle est aussi une riposte en réaction à l'arrestation de la suffragette Emmeline Pankhurst. Dans ses heurts avec 
les groupes de suffragettes, la police britannique fait face à un soulèvement de femmes prêtes à l'engagement physique dans la bataille pour leurs droits politiques, donnant lieu à de véritables affrontements attisés par le "Cat and Mouse Act $^{14}$ ", un phénomène dont l'ampleur est alors sans égal en France. Richardson explique juste après son acte qu'elle dénonce l'injustice qu'il y a à laisser mourir une femme réelle, vivante, alors que l'on célèbre et protège les corps féminins idéalisés des chefs-d'œuvre de l'histoire de l'art, soumis aux regards et sans potentialité d'action ${ }^{15}$. Elle fait une analogie entre l'illusion d'une chair représentée en peinture - le corps de la Vénus - et une vraie chair, celle d'Emmeline Pankhurst, alors emprisonnée et en pleine grève de la faim ${ }^{16}$. S'en prendre à la Vénus de la National Gallery c'est, selon Richardson, attirer l'opinion publique sur des pratiques carcérales qui consistent à nourrir de force les suffragettes en grève de la faim, et répondre à une violence physique par une altération matérielle ${ }^{17}$. Si son intention est connue et relayée par la presse, des journalistes y voient un geste guidé par la jalousie des suffragettes anglaises envers Vénus, déesse de l'amour, de la beauté et symbole d'un idéal féminin. L'historienne Karen M. Offen identifie les mécanismes d'actions caractéristiques des suffragettes du WSPU en précisant qu'elles renoncent à une "bienséance féminine ${ }^{18}$ » et cite Pankhurst : « Nous avons essayé de nous conduire en femmes, nous avons essayé d'utiliser notre pouvoir féminin, et nous avons constaté que cela ne sert à rien $[. . .]^{19}$. »

5 La visibilité médiatique de ces troubles à l'ordre public donne lieu à des commentaires outre-Manche, où l'on oppose au désordre des suffragettes anglaises la prétendue subtilité des féministes en France ${ }^{20}$. L'image des militantes françaises suivant la voie légale différait de celle des suffragettes anglaises pénétrant avec fracas dans la sphère masculine, allant au-delà des limites supposées de leur sexe. En France la plupart des suffragistes ${ }^{21}$ qui concentrent leurs campagnes sur l'accès au droit de vote en priorité n'emploient que très rarement des actions publiques aussi violentes que celles des suffragettes anglaises du WSPU. La "politique de l'assaut " proposée dès les années 1880 par Hubertine Auclert (1848-1914), fondatrice de Ligue française pour le droit des femmes (LFDF), est minoritaire dans le féminisme français ${ }^{22}$. Si Auclert réussit à mobiliser la presse en faisant des coups d'éclat dans l'espace public, ils sont relativement inoffensifs par rapport aux démonstrations organisées des manifestations de masse des suffragettes du WSPU qui radicalisent leurs modes opératoires dans les années $1910^{23}$. Le 17 mars 1914, quelques jours après l'attaque de la Vénus de la National Gallery, Auclert, invitée à commenter l'évènement pour «ceux que font frémir les suffragettes britanniques », déclare au journal La Vie Féminine : « est-ce qu'on prend nos Ligueuses pour des violentes? Point ! [...] Nous agissons par les affiches, par l'aide que veut bien nous donner la Presse, et par des réunions et des conférences ${ }^{24} »$. Ce détachement montre bien la mesure des moyens employés par le WSPU, violents et inédits. Les moments d'empoigne avec la police font l'objet de commentaires ou de caricatures, comme ce dessin publié en 1910 (fig. 1) dans le journal satirique Le Rire ${ }^{25}$. Le policeman brandit le manche de sa matraque, presque un présage de celui du hachoir qui troue quatre ans plus tard la toile de la Vénus, et s'apprête à porter un coup sur la tête de la suffragette à l'expression féroce, montrant (ou serrant) les dents, les poings prêts à riposter. La légende sous forme de dialogue entre les deux protagonistes rappelle que la place de la femme, d'une lady, est de plaire à l'homme, en souriant et en lui étant agréable, et non de rugir. Cette récurrence des attaques misogynes sur l'apparence des dissidentes n'est pas sans rappeler celles formulées régulièrement dans ces années à l'encontre des femmes célibataires ou des femmes artistes ${ }^{26}$. 
Fig. 1

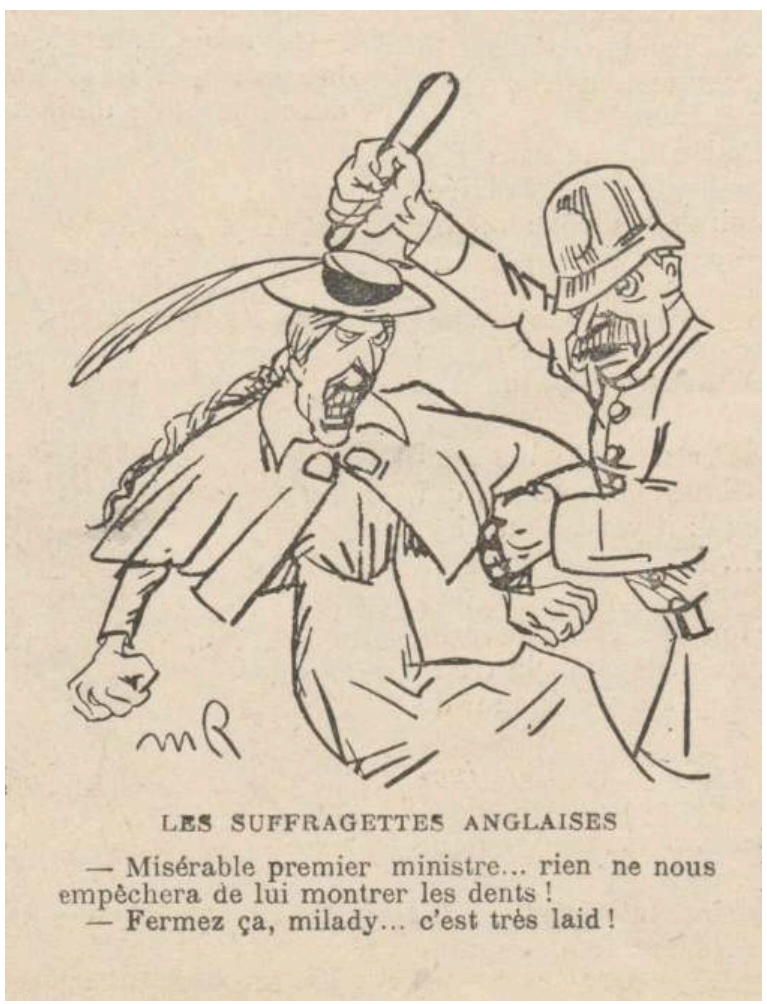

Radiguet, «Les suffragettes anglaises », Le Rire - journal humoristique -, 10 décembre 1910, p. 10, dessin satyrique, Paris.

(c) Bnf (c) Gallica

Les images de femmes violentes agissant en dehors de l'ordre social établi existent déjà dans les représentations de figures féminines vétérotestamentaires. Celles-ci ont été analysées par les historiennes de l'art féministes dont Griselda Pollock qui a étudié dans son ouvrage Differencing the Canon la fabrique des images de ces héroïnes en s'appuyant sur les sujets des tableaux d'Artemisia Gentileschi (1593-1653) ${ }^{27}$. L'une des iconographies les plus commentées de son œuvre est celle de Judith décapitant Holopherne, qui éclipse d'autres épisodes de l'Ancien Testament comme l'histoire de Jaël tuant Sisera, sujet d'un tableau (fig. 2) de Gentileschi en 1620. Dans le Livre des Juges, l'héroïne juive recueille dans sa tente un général ennemi, Sisera, puis le tue dans son sommeil en enfonçant dans sa tempe un clou de tente avec un marteau, provoquant ainsi la victoire d'Israël sur l'armée Cananéenne. L'épisode figure dans La gallerie des femmes fortes de Pierre Le Moyne au XVII siècle :

« [...] Jaël tira sans bruit un des clous dont sa Tente était suspendue, et à coups de marteau le lui mit si avant dans la tête, qu'il la lui perça de part en part, et entra dans la terre avec son sang et son âme. Cette Femme valut toute une Armée ; et un clou en sa main, fit ce que dix mille lances et autant d'épées, n'avaient pu faire ${ }^{28}$. » 
Fig. 2

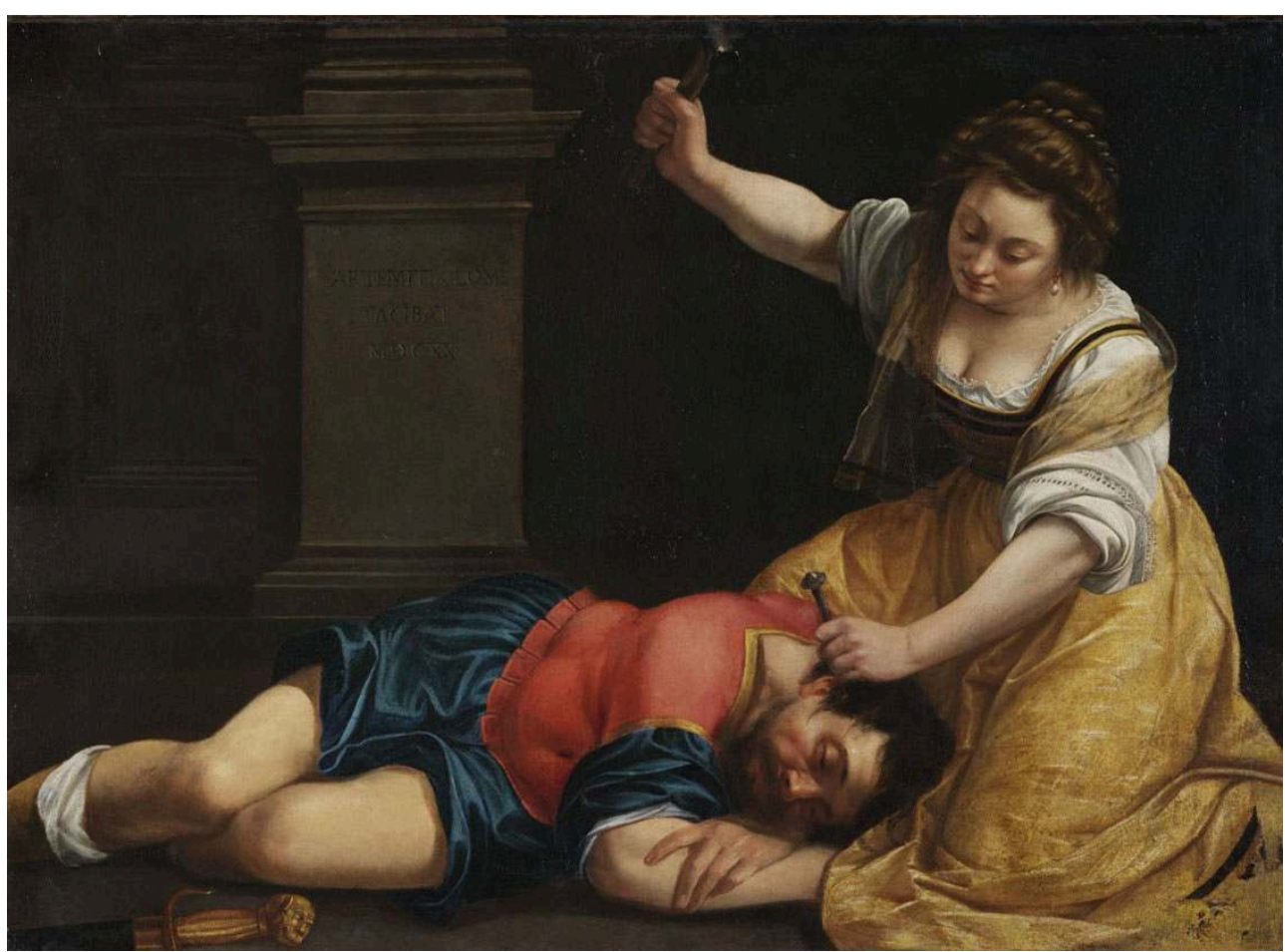

Artemisia Gentileschi, Jaël et Sisera, 1620, huile sur toile, Budapest, Musée des Beaux-arts. (C) $\mathrm{CC}$

7 Le moment choisi par Artemisia Gentileschi est celui, caractéristique et dramatique, du geste de Jaël brandissant son marteau au-dessus de la tempe de Sisera. Dans son tableau Jaël et Sisera, la peintre isole le groupe dans un environnement neutre et dépouillé, où le calme du sommeil et de la composition précède le massacre, offrant une image beaucoup plus sereine que le meurtre d'Holopherne par décapitation. Dans son essai, Griselda Pollock mentionne l'étude de Mieke Bal qui analyse l'iconographie de l'histoire de Judith, et dans une moindre mesure celle de Jaël, dans les tableaux de Rembrandt. Son ouvrage Reading Rembrandt, Beyond the Word-Image Opposition, Bal démontre qu'un dessin de Rembrandt figurant Jaël munie d'un marteau et s'apprêtant à planter le clou dans la tête de Sisera correspond à l'image d'" un sculpteur au travail ${ }^{29}$ ". Le geste du bras de Jaël donnant le coup fatal à Sisera, est similaire à celui d'un sculpteur - d'une sculptrice - achevant sa création ou détruisant son œuvre ${ }^{30}$. Peut alors s'opérer le glissement d'un geste meurtrier dans l'histoire de Jaël vers le geste créateur d'une œuvre d'art, où l'exécutante est une femme brandissant le marteau de la sculpture. Transposée dans la sphère artistique, cette formule iconologique est semblable à l'allégorie de la sculpture fixée par l'ouvrage de Cesare Ripa ${ }^{31}$ puis largement utilisée au XVIII ${ }^{\mathrm{e}}$ siècle, où la représentation de la Sculpture oscille entre une femme tenant dans ses mains la pointe et le maillet ${ }^{32}$ et les représentations du mythe de Pygmalion $^{33}$.

\section{Pointes et marteaux, coups et blessures : des enjeux de (la) taille}

La formule iconographique de la pointe et du marteau de Jaël se retrouve aussi dans des images de sculptrices, de façon plus ou moins menaçante et destructrice en fonction 
des images et du discours qui l'accompagnent. En 1789, la sculptrice britannique Anne Seymour Damer (1748-1828) est caricaturée par W. M. Holland (fig. 3), dans son atelier en présence de statues et d'une jeune fille de dos. Elle est assise, porte une longue robe resserrée au niveau de la taille et avec ses mains gantées, elle effectue un geste gracieux et élégant ; sa main droite est armée d'un maillet brandi au-dessus de sa tête, destiné à s'écraser sur la pointe qu'elle tient dans sa main gauche, dirigée vers le postérieur d'une statue d'Apollon. La pointe, placée au centre de la composition et vers laquelle convergent les regards des deux figures féminines, laisse présager une blessure que l'historienne de l'art Alison Yarrington analyse comme la représentation d'une castration par l'arrière ${ }^{34}$. Dans l'image, l'Apollon, une sculpture attribuée à Damer ${ }^{35}$ donnant son titre au dessin The Damerian Apollo, peut être interprété comme le vrai corps d'un homme, subissant lui aussi une mutilation qui altèrerait directement sa masculinité. Le caractère humoristique - ou menaçant - de la scène réside d'ailleurs dans cette ambiguïté du corps masculin qui, bien que plus grand que nature, semble à la merci de la sculptrice.

Fig. 3

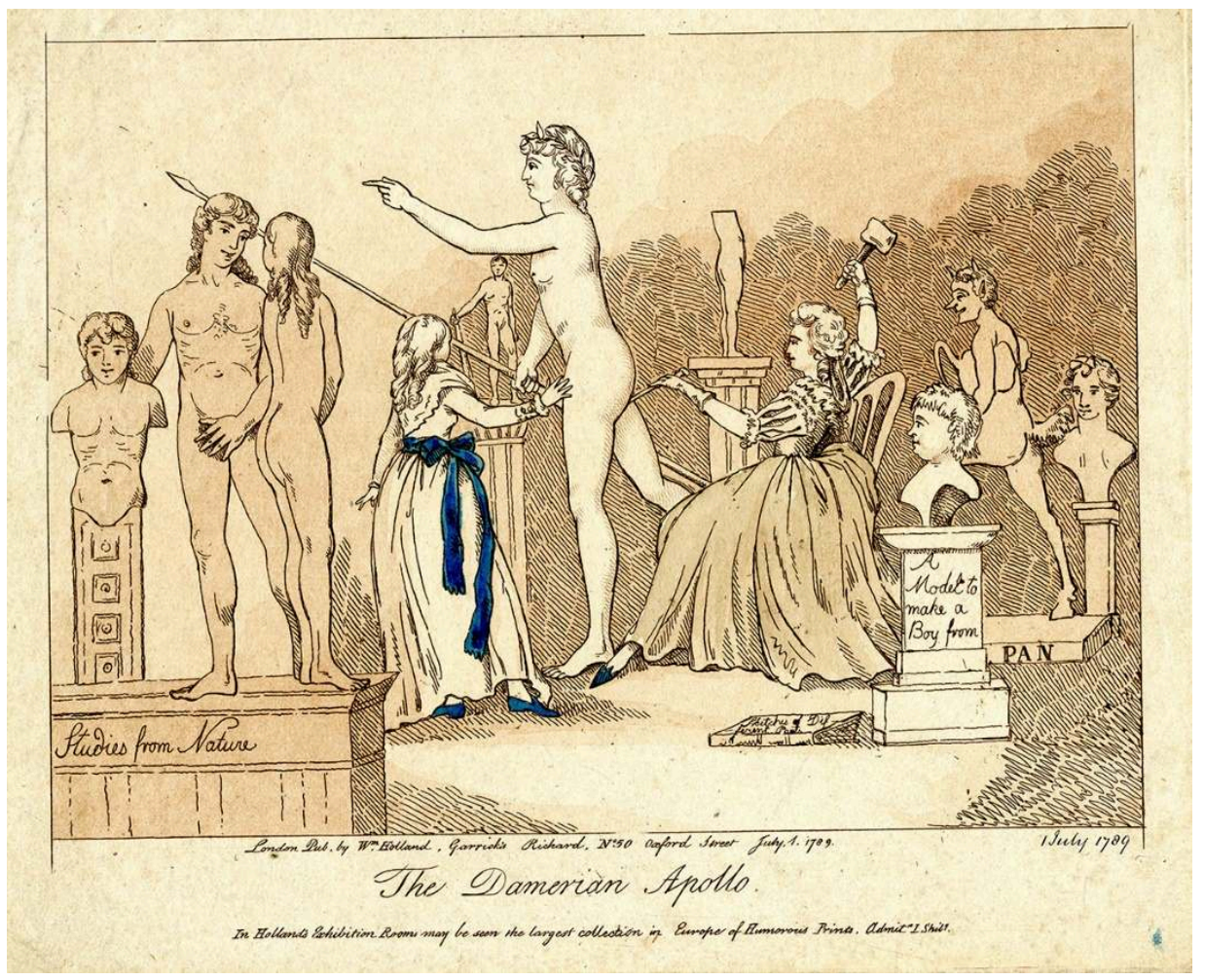

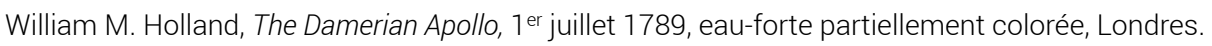

(C) Trustees of British Museum

9 Nous proposons de rapprocher la charge violente de la caricature anglaise d'une gravure sur bois représentant Michel-Ange au travail penché au-dessus d'une sculpture de femme allongée, dans une position correspondant à l'antique Ariane endormie ${ }^{36}$. Sa poitrine est menacée par le ciseau du sculpteur qui s'apprête à y enfoncer la pointe à l'aide du maillet, reproduisant le geste ample de Damer, semblable à l'agression physique d'un vrai corps ${ }^{37}$. Les deux scènes, où la sculptrice et le sculpteur attaquent des parties de corps très spécifiques et particulièrement genrées, montrent la violence 
potentielle des images de la pratique artistique. Yarrington a aussi interprété le geste castrateur de Damer dans une perspective plus symbolique, comme une "force destructrice au sein des Arts représentés par Apollon ${ }^{38}$ ». Damer est en effet à cette époque à la recherche de commandes publiques, ce qui la place en compétition avec d'autres sculpteurs ${ }^{39}$. Armée de son ciseau et de son marteau, elle représente alors une menace dans le domaine artistique, réveillant les peurs de ses concurrents masculins, mis en danger par sa présence et sa reconnaissance institutionnelle dont ils avaient jusqu'alors le monopole ${ }^{40}$. Une telle violence de l'image, qui fait explicitement état du rapport de pouvoir entre féminin et masculin, ne se retrouve pourtant que très rarement à la fin du XIXe siècle, malgré l'intensification et l'organisation de la lutte féministe et sa progression dans les territoires masculins de l'art et de la politique. En effet, depuis les années 1880 au moins le monde des arts n'est pas hermétique à ces questions sociales et en France, des revendications politiques sont portées par des femmes artistes demandant à bénéficier d'une formation égale à celle des hommes à l'École des beaux-arts, ce qu'elles obtiennent à partir de 1897. Dans le domaine artistique l'un des évènements marquants de cette progression des femmes artistes dans les lieux d'enseignement est la victoire de la sculptrice Lucienne Heuvelmans (1881-1944) qui remporte en 1911 le premier Prix de Rome, récompense ultime de l'École des beaux-arts auquel les femmes ont le droit de concourir depuis $1903^{41}$. Elle devient ainsi la première femme artiste à entrer à la Villa Médicis et y inaugure la mixité. En France, le succès de Lucienne Heuvelmans, qui ne semble pas avoir fait partie de groupes militants, est reçu comme une victoire politique par la presse féministe ${ }^{42}$ et généraliste. Dans un article dédié à la sculptrice par Georges Montorgueil, époux de la sculptrice Laure Coutan-Montorgueil, elle-même membre de l'UFPS, le journaliste recrée la filiation avec les combats d'Hélène Bertaux, décédée en 1909, et déploie un discours guerrier transformant l'accomplissement individuel en exploit collectif :

«[Lucienne Heuvelmans] aura dans l'histoire de l'art une place à part: celle du vaillant qui, le premier, entre par la brèche ouverte. Mais cette brèche, on oublie peut-être un peu celle qui l'ouvrit. Si Mlle Heuvelmans va à Rome, c'est parce que Mme Léon Bertaux lui en a permis l'accès [...] Fermée aux femmes, [l'École des beaux-arts] était la citadelle qu'il importait de conquérir. Mme Léon Bertaux appelle à la rescousse ses compagnes, ses émules. Elle les groupe pour l'engagement suprême. Quand elle les harangue, on croirait entendre le jeune Bonaparte à la veille d'Arcole, montrant à ses soldats le chemin des conquêtes. N'était-ce pas aussi, pour l'intrépide stratège, sa campagne d'Italie ? [...] La Villa Médicis, en 1903, enfin, se rend ${ }^{43}$.»

10 Montorgueil évoque aussi plus loin dans ce même texte les "petits poings de femme " de Bertaux qui tambourinèrent aux portes des institutions et, au risque de réduire l'entreprise collective de l'UFPS à une initiative individuelle, il fait de Bertaux une incarnation de cette lutte pour l'entrée aux beaux-arts et à la Villa Médicis. Cette véhémence qui ne se retrouve que très partiellement dans la culture visuelle de l'époque témoigne d'une volonté de conciliation, d'une stratégie du compromis pour faire accepter l'institutionnalisation des femmes artistes.

11 Grâce à la photographie, l'image de l'artiste - femme et homme - au travail se diffuse au XIX ${ }^{e}$ siècle ${ }^{44}$, et bien que les représentations de la peintre soient plus répandues que celles de la sculptrice, celles-ci existent et circulent. Dans Femina, revue bimensuelle illustrée fondée en 1901 par Pierre Lafitte, les femmes artistes sont domestiquées. Dans le Monde illustré: sculptrices peu montrées, des portraits de groupe d'ateliers de 
femmes peintres. Parmi les journaux destinés à un lectorat féminin et contrairement aux publications féministes comme La Citoyenne créée par Hubertine Auclert ou La Fronde fondée par Marguerite Durand, Femina se veut apolitique et promeut une vision essentialiste de la féminité ${ }^{45}$. Fondée par Pierre Lafitte en 1901, la revue a aussi pour particularité d'être richement illustrée de reproductions de photographies qui accompagnent des courts entretiens, dont certains avec des femmes artistes. L'historienne de l'art Charlotte Foucher Zarmanian en étudiant les discours genrés sur les femmes artistes dans la presse vers 1900, démontre que les revues comme la Vie Heureuse et Femina, destinées aux bourgeoises, véhiculent des valeurs familiales conservatrices et des préoccupations éloignées des combats féministes ${ }^{46}$. Lors de la victoire de Lucienne Heuvelmans en 1911, Femina reproduit certes en pleine page la photographie de la sculptrice accoudée à une sellette vide devant un fond peint censé représenter la Villa Médicis, mais les quelques lignes de l'article qu'elle illustre précisent bien : « Les femmes - et non le féminisme, comme l'écrivent incorrigiblement beaucoup de nos confrères - viennent de remporter une éclatante victoire sur la $\operatorname{Tradition}^{47}$ ». Déjà quelques années plus tôt, un journaliste de Femina souligne à propos de son travail de sculptrice qu'il est remarquable « car la sculpture est un art qui exige une énergie physique plus grande, un travail plus rude, et par là même semble plus audelà des forces de la femme ${ }^{48}$ » et continue ainsi à véhiculer les stéréotypes de genre sur la place - inférieure à celle des hommes - que doivent occuper les femmes dans les beaux-arts puisque "[leurs] doigts agiles, habitués à tenir l'aiguille, se prête[raie]nt plus facilement aux menues opérations, à l'emploi des petits outils, à la succession de mouvements imperceptibles ${ }^{49}$ ». Ce sont ces "menues opérations" qui sont mises en évidence dans la photographie (fig. 4) qui montre Claudel, absorbée par son geste de son court marteau, dirigeant la petite pointe vers le groupe Vertumne et Pomone, dans un article sur les artistes femmes au Salon de 1903. Elle utilise les codes de représentation féminins, loin des réalités de l'atelier de sculpture, avec sa robe corsage apprêtée, sans salissures, un flot dans les cheveux, qui correspond à l'image de la «femme artiste en modèle attractif $\mathrm{f}^{50}$ ", qui doit plaire à un magazine destiné à un lectorat de bourgeoises. Dans la photographie de Claudel, l'association du marteau et de la pointe dans un geste très étriqué, contraint par un cadrage très rapproché, façonne une image de l'artiste femme en sculptrice minutieuse, qui porte aussi le message d'une artiste restant dans les limites de son genre, loin de la virulence de l'iconographie de Jaël. Son geste et ses outils perdent de leur menace potentielle, amoindrissent le pouvoir agressif - voire subversif - de l'image. La sculpture peut être décrite comme un art viril mais les représentations dans Femina de sculptrices respectent les assignations de genre afin de rendre acceptable leur nouvelle place - et leurs nouvelles victoires - dans le domaine des beaux-arts. C'est aussi le cas pour les féministes, comme le rappelle l'historienne Florence Rochefort d'ans une autre caricature célèbre d'Hubertine Auclert en 1880 par Albert Robida pour le journal La Caricature, dont le numéro spécial «Le Coup d'état féminin ${ }^{51}$ » la représentant une bouteille de vitriol à la ceinture en train de brandir le drapeau des droits de la Femme ${ }^{52}$. Les armes sont bien "féminines", ainsi que leurs costumes, qui apportent un contraste avec la puissance de leur révolte jusqu'au comique $^{53}$. Ces images cohabitent pourtant avec d'autres, annonciatrices d'un changement social bien en marche: la photographie de la sculptrice Jane Poupelet (1874-1932) publiée dans une édition du New York Times en $1916^{54}$ offre l'image d'une artiste volontairement androgyne, dont la blouse de travail ample efface les formes $\mathrm{du}$ corps, les cheveux ramassé en chignon pour dégager le visage, elle tient un instrument 
de mise au point, indiquant sa capacité à réaliser l'aspect technique de sa sculpture, un procédé de mise en scène similaire dans son intention à celui de Camille Claudel dans le cliché de 1903. Cependant, dans ce portrait posé avec son œuvre Imploration, Poupelet semble beaucoup plus à l'aise, faisant preuve d'une maîtrise de son image qui se veut peut-être plus décontractée, puisqu'elle a à la bouche une discrète mais pourtant bien visible cigarette ${ }^{55}$ avant que celle-ci ne devienne l'attribut de la garçonne et de l'émancipation féminine des années 1920.

Fig. 4

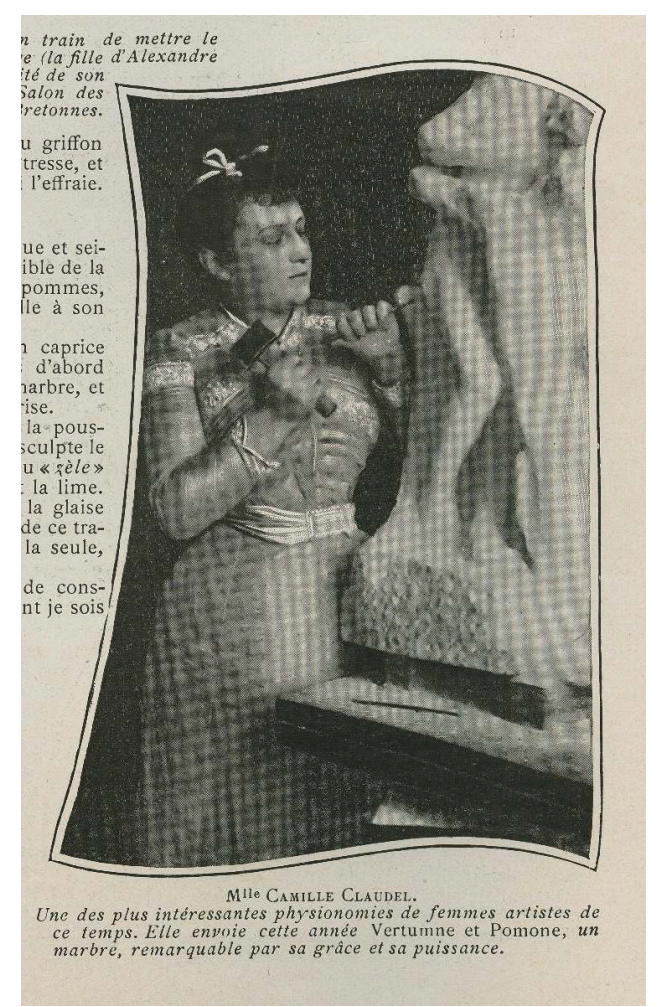

Anonyme (Simons), « Mlle Camille Claudel », Femina, Paris, n 55, $7^{\text {er }}$ mai 1903, pp. 520-521,

photographie accompagnant l'article de Gabrielle Reval, « Les Artistes femmes au Salon de 1903 », Paris.

(C) Agence photographique du musée Rodin

\section{Conclusion}

En 1914, un journal français compare la mutilation au hachoir de la Vénus à la National Gallery à une "opération culinaire, [un] dépeçage" puisque, selon l'article, Mary Richardson « découp[e] [la toile] avec un hachoir - comme un homard à l'Américaine $[. . .]^{56} »$. Le hachoir, évoque celui-ci du boucher mais aussi la cuisine du foyer, et c'est d'ailleurs ce territoire domestique, connoté féminin par excellence, qu'évoque Le Hachoir, l'œuvre de Niki de Saint Phalle (1930-2002) de 1960 où la cohabitation du rouleau à pâtisserie et du hachoir, dans une composition aux allures d'arma christi, contient ce que l'historienne de l'art Déborah Laks qualifie de «violence symbolique des ustensiles quotidiens $s^{57}$ ». Cette mise en scène de la violence se retrouve aussi dans ses performances du début des années 1960, qui précèdent les Nanas : en 1962, Niki de Saint Phalle tire ainsi sur une autre Vénus, celle de Milo cette fois-ci, pour la pièce The 
Construction of Boston. Le geste peut être interprété comme un rejet des canons traditionnels de l'art, qu'il s'agisse des proportions ou de l'idéal. Un an auparavant, en 1961, Saint Phalle s'autoproclame «terroriste artistique ${ }^{58}$ » et tire à la carabine sur des surfaces où sont agglomérés des objets et des poches remplies de peintures qui se crèvent sous l'effet des balles, faisant couler la couleur. Certaines performances sont filmées, et retransmises à la télévision, montrant ce que l'historienne de l'art Catherine Gonnard appelle un " jeu ambigu ${ }^{59}$ » entre l'apparente inoffensivité de l'artiste parfois habillée en jeune fille de bonne famille, et la jubilation qui accompagne sa violence quand elle tire sur l'OAS en pleine guerre d'Algérie en 1962, ou encore sur le SIDA en $1987^{60}$.

\section{NOTES}

1. D. Henry-Asselin, « Une Femme artiste », La Presse, 10 mars 1905, n. p.

2. Un aspect que la lecture de sa correspondance vient contredire. Voir Camille Claudel, Correspondance, édition d'Anne Rivière et Bruno Gaudichon, $3^{\mathrm{e}}$ édition revue et augmentée, Paris, Gallimard, 2014.

3. Paul-Louis Rinuy, «Le statuaire et le marbre à la fin du XIX ${ }^{\mathrm{e}}$ siècle », cat. d'exp., Rodin, la chair, le marbre, sous la direction d'Aline Magnien, Musée Rodin, 2012-2013, Paris, Hazan, 2012, pp. 29-40.

4. María López Fernández, « Derrière le mythe de Camille Claudel : la construction d'une image », cat. d'exp., Camille Claudel, 1864-1943, Musée odin, 2013-2014, Paris, Musée Rodin; Madrid, Fundacion Mapfre, 2007, pp. 66-79.

5. Jean-François Corpataux, Le Corps à l'œuvre : sculpture et moulage au XIX ${ }^{e}$ siècle, Genève, Librairie Droz, 2012, pp. 229-232.

6. Catherine Chevillot, La Sculpture à Paris : 1905-1914, le moment de tous les possibles, Vanves, Hazan, 2017, pp. 114-116.

7. Voir Victor I. Stoichita, L'Effet Pygmalion. Pour une anthropologie historique des simulacres, Genève, Librairie Droz, 2008.

8. Encore au esiècle, en 1938, le critique Louis Vauxcelles écrit dans son article «Femmes sculpteurs d'hier et d'aujourd'hui ", (Le Monde illustré, n 4210, 24 septembre 1938, n. p.), que la sculpture est «ce rude métier [...] [pour lequel] une singulière résistance physique est requise ». En reprenant cette idée largement répandue depuis le siècle précédent, l'auteur justifie ainsi le nombre moins important de sculptrices par rapport aux femmes peintres par les difficultés physiques qu'auraient les femmes à sculpter.

9. Sur l'entrée des femmes à l'École des beaux-arts, voir Marina Sauer, L'Entrée des femmes à l'École des beaux-arts, 1880-1923, Paris, ENSBA, 1991.

10. Voir Sophie Moiroux, «L'image empreinte d'intentions. La "Vénus tailladée". Considérations sur un acte d'iconoclasme", Images Re-vues, n² 2, 2006 ; cat. d'exp., Art under Attack: Histories of British Iconoclasm, sous la direction de Tabitha Barber, Stacy Boldrick, Tate Britain, Londres, 2013-2014, Londres, Tate Publishing, 2013 ; Dario Gamboni, The Destruction of Art: Iconoclasm and Vandalism Since the French Revolution, Londres, Reaktion Books, 2018.

11. Helen Harden Chenut, "L'esprit antiféministe et la campagne pour le suffrage en France, 1880-1914 », Cahiers du Genre, 2012/1 (nº 52), pp. 51-73, paragraphe 7. 
12. Krista Cowman, "Suffragette attacks on art, 1913-1914», Suffrage and the Arts: Visual Culture, Politics and Enterprise, Londres, Bloomsbury Visual Arts, 2019, pp. 185-202.

13. Nous employons ici le mot qui revient le plus fréquemment dans la presse française de l'époque. Dans son article, Sophie Moiroux indique que l'arme de Richardson n'est pas un hachoir à proprement dit, mais «un petit couteau». Elle indique dans une note que Richardson a rapporté dans son autobiographie (Laugh a Defiance, Londres, George Weidenfeld \& Nicholson, 1953, p. 166) qu'elle s'est servi d'une axe, que l'on peut traduire par « hache ». L'autrice cite dans une autre note le rapport des experts, publié dans l'édition du Time du 11 mars 1914, où apparaît le mot chopper, lequel désigne un « couperet » ou un « hachoir".

14. Temporary Discharge for Ill-Health, mesure votée en 1913 par le Parlement britannique afin de lutter contre les grèves de la faim entreprises par les suffragettes emprisonnées, constituant à les relâcher pour quelques jours lorsque fragilisées par leurs grèves, puis à les emprisonner à nouveau quelques jours plus tard, une fois qu'elles ont repris des forces et ne risquent plus de mourir en prison.

15. "The deed exploited the double nature of the sign: as signified, it could receive 'wounds' meant to expose and maybe to avenge the pains inflicted on the imprisoned feminists, while as signifier it enabled the dismissal of moral judgements passed on the destruction of what was 'only a picture' ». D. Gamboni, op. cit. note 10, p. 119.

16. Chloe Ward, "Images of empathy : Representations of force feeding in Votes for Women ", Suffrage and the Arts: Visual Culture, Politics and Enterprise, Londres, Bloomsbury Visual Arts, 2019, pp. 249-272.

17. "The public did not care about this living woman, but I knew they would care about cut up a laid of canvas of both financial and artistic value." "Mary Richardson ", The Woman's Hour, BBC 4, 12 Septembre 1957, 3'58 mn.

18. Karen M. Offen, Les Féminismes en Europe 1700-1950, Rennes, Presses universitaires de Rennes ; Dinan, Terre de Brume, coll. Archives du Féminisme, 2012, p. 293.

19. Emmeline Pankhurst, Votes for Women, $\mathrm{n}^{\circ} 34,29$ octobre 1908, p. 81, cité dans K. M. Offen, op. cit. note 18 .

20. « [Elles] ont adapté Vénus au monde moderne en habillant sa nudité, et, sous le nom de Mode, ne craignent point de rendre ce qui lui est dû à l'éternelle déesse de la séduction féminine ", Pierre Bonhomme, «La Vénus Mutilée », Le Radical, 11 mars 1914, p. 2.

21. Cette différence entre les militantes féministes qui réclament le droit de vote s'observe aussi dans l'emploi des mots : la «suffragiste » est une version modérée de la «suffragette », celle-ci étant « une guerrière qui a entrepris la conquête armée des droits politiques de la femme » selon Jane Misme (« Les suffragistes en marche » L'Opinion, 19 juillet 1913), citée dans Laurence Klejman et Florence Rochefort, L'Égalité en marche, le féminisme sous la Troisième République, Paris, Presses de la Fondation nationale des sciences politiques, Des femmes, 1989, p. 267.

22. Sur Hubertine Auclert, voir Édith Taïeb, "Hubertine Auclert, féministe "intégrale" ", Les Féministes de la première vague, Rennes, Presses universitaires de Rennes, collection Archives du féminisme, 2015, pp. 31-45. Voir aussi Steven C. Hause, Hubertine Auclert, pionniere du féminisme textes choisis, Saint-Pourçain-sur-Sioule, Bleu autour, 2007.

23. K. M. Offen, op. cit. note 18, pp. 292-293.

24. André Arnyvelde, «Une visite à la Mère des Suffragettes françaises ", La Vie Féminine, 17 mars 1914, p. 2.

25. Dessin de Radiguet, « Les suffragettes anglaises », Le Rire, 10 décembre 1910, n. p.

26. Charlotte Foucher Zarmanian, Créatrices en 1900. Femmes artistes en France dans les milieux symbolistes, Paris, Mare \& Martin, 2015, pp. 47-67.

27. Voir plus particulièrement le chapitre «The Female Hero and the Making of a Feminist Canon »: Griselda Pollock, Differencing the Canon: Feminist Desire and the Writing of Art's Histories, Londres et New York, Routledge, 1999, pp. 96-127. 
28. Pierre Le Moyne, La gallerie des femmes fortes, Paris, La Compagnie des Marchands Libraires du Palais, $5^{\mathrm{e}}$ édition, 1665 , p. 39. L'orthographe a été modernisée.

29. «As an anti-Pygmalion, we see here a visual artist, a sculptor at work on something that can at any moment become alive ». Mieke Bal, Reading Rembrandt: Beyond the Word-Image Opposition, Cambridge, New York, Cambridge University Press, 1991, p. 297. Le dessin de Rembrandt est visible en ligne sur le site du Rijksmuseum www.rijksmuseum.nl/en/search/objects ? $q=$ jael + rembrandt $\& p=1 \& p s=12 \&$ st $=$ Objects\&ii $=0 \# /$ RP-T-1930-8,0 [16/07/2020].

30. "The suspenseful narrative of murder (e.g. will he wake up before he is killed?) is replaced by the equally suspenseful representation (e.g., is the work destructive or creative?) ", Idem, ibidem.

31. Cesare Ripa, Iconologie, ou la Science des emblèmes, devises, etc., qui apprend à les appliquer, dessiner et inventer ... augmentée d'un grand nombre de figures avec des moralités, tirées la plupart de César Ripa, Amsterdam, A. Braakman, 1698, p. 542.

32. Mary D. Sheriff, Moved by Love: Inspired Artists and Deviant Women in Eighteenth-Century France, Chicago, University of Chicago Press, 2004, pp. 159-189.

33. V. I. Stoichita, op. cit. note 7.

34. Alison Yarrington, «The Female Pygmalion : Anne Seymour Damer, Allan Cunningham, and the Writing of a Woman Sculptor's Life », The Sculpture Journal, $\mathrm{n}^{\circ}$ 1, 1997, p. 34.

35. Marjan Sterckx, «The Invisible "Sculpteuse" : Sculptures by Women in the Ninteenth Century Urban Public Space - London, Paris, Brussels ", Nineteenth-Century Art Worldwide, $\mathrm{n}^{\circ}$ 2, 2008.

36. Anonyme, Michel-Ange au travail, gravure sur bois (dans Sigismondo Fanti, Triompho di Fortuna, Venise, 1527), reproduit dans J.-F. Corpataux, ill. 103, p. 231.

37. Dans son ouvrage, J.-F. Corpataux met en rapport Michel-Ange et Géricault, reproduisant le groupe sculpté de ce dernier, Nymphe et satyre, 1818, conservé au musée des beaux-arts de Rouen (p. 232). Cette sculpture de Géricault dans laquelle un satyre semble s'attaquer à une nymphe qui le repousse, a été analysée par Régis Michel comme une agression sexuelle violente. Voir Régis Michel, « L'Art du viol », Mouvements, n² 20, 2002, pp. 84-97.

38. "Thus Damer, in her feminine attire, is identified as a destructive force within the Arts represented by Apollo, a kind of 'inverted' muse ». A. Yarrington, art. cité, note 34. Traduction de l'autrice.

39. M. Sterckx, op. cit. note 35.

40. Il s'agit d'une rhétorique utilisée des décennies plus tard par certains Académiciens pour qualifier l'entrée à la Royal Academy d'artistes femmes dans les années 1860. Voir George Dunlop Leslie, The Inner Life of the Royal Academy, Londres, John Murray Albemarle Street. W., 1914, pp. 42-43.

41. M. Sauer, op. cit. note 9, p. 36.

42. En 1911, la revue annuelle L'Action féminine, organe de presse officiel du Conseil national des femmes françaises (CNFF) dirigé par Mme Avril de Sainte-Croix, publie une brève dans sa rubrique qui compile les actualités du féminisme dans le monde et tire les conséquences de «Victoire pour le féminisme»: «À partir de ce moment, l'art ne connait plus de sexes». Anonyme, "Actualités ", L'Action féminine, bulletin officiel du Conseil national des femmes françaises, 1911, p. 316.

43. Georges Montorgueil (Octave Lebesgue), «L'Assaut de la Villa Médicis », Le Grand Écho du Nord et du Pas-de-Calais, 5 août 1911, p. 1.

44. Rachel Esner, « Nos artistes chez eux. L'image des artistes dans la presse illustrée », L'artiste en représentation. Images des artistes dans l'art du XIX ${ }^{e}$ siècle, Alain Bonnet, Hélène Jagot (dir.), Lyon, Fage éditions, 2013, p. 138-149. Voir aussi Pierre Wat (dir.), Portraits d'ateliers. Un album de photographies fin de siècle, Grenoble, ELLUG, INAH, MSH Alpes, 2013.

45. H. Harden Chenut, art. cité note 11, paragraphe 14, en ligne https://www.cairn.info/revuecahiers-du-genre-2012-1-page-51.htm\#re2no2 
46. C. Foucher Zarmanian, «Les femmes artistes sous presse. Les créatrices vues par les femmes critiques d'art dans la presse féminine et féministe en France autour de 1900 ", Sociétés \& Représentations, 2015/2 ( $\mathrm{N}^{\circ} 40$ ), pp.111-127, en ligne URL: https://www.cairn.info/revuesocietes-et-representations-2015-2-page-111.htm consulté le 9/10/19.

47. H. A., « Une femme remporte le prix de Rome », Femina, 15 août 1911, p. 446.

48. Laurent Drevet, « En loge », Femina, 15 août 1906, p. 366.

49. Léon Lagrange, « Du rang des femmes dans les arts », Gazette des beaux-arts, $n^{\circ} 43,1^{\text {er }}$ octobre 1860, p. 36.

50. C. Foucher Zarmanian, op. cit. note 46.

51. "La Grande Société secrète des droits de la femme lève l'étendard sacré de la révolte... ", $L a$ Caricature, 16 octobre 1880.

52. Sur ce sujet voir Karine Salomé, Vitriol, les agressions à l'acide du XIX siècle à nos jours, Ceyzérieu, Champ Vallon, 2020.

53. Florence Rochefort, «L'Antiféminisme à la Belle Époque, une rhétorique réactionnaire », Un siècle d'antiféminisme, Paris, Fayard, 1999, p. 140.

54. "Jane Poupelet ou la "La beauté dans la simplicité" ", cat. d'exp., Jane Poupelet (1874-1932), la beauté dans la simplicité, sous la direction de Bruno Gaudichon et Anne Rivière, Roubaix 2005-2006, Paris, Gallimard, 2005, p. 45.

55. Cet accessoire est loin d'être anecdotique puisqu'il est associé aux féministes qui s'approprient les codes masculins, dont le pantalon et la bicyclette, comme on peut le voir dans une caricature du journal Le Grelot en 1896 («Revendications féminines », Le Grelot, 19 avril 1896), reproduite dans Tamar Garb, Sisters of the Brush, Women's Artistic Culture in Late Nineteenth-Century Paris, New Haven, Londres, Yale University Press, 1994, p. 118.

56. Saint-Roman, « Les Musées de Londres », L'Univers, 19 mars 1914, p. 1.

57. Déborah Laks, Des déchets pour mémoire. L'utilisation de matériaux de récupération par les nouveaux réalistes (1955-1975), Dijon, Presses du réel, 2017, p. 162.

58. Amelia Jones, «Wild Maid, Wild Soul, a wild wild weed : les féminisés féroces de Niki de Saint Phalle vers 1960-1966 ", cat. d'exp., Niki de Saint Phalle (1930-2002), sous la direction de Camille Morineau, Paris, Réunion des musées nationaux-Grand Palais, 2014, pp. 156-163.

59. Catherine Gonnard, « Niki de Saint Phalle, figure de proue de l'avant-garde à la télévision », ibid, p. 112 .

60. Laurence Bertrand Dorléac, "Vivre pour vivre. Les conditions d'une autre politique ", cat. d'exp. Niki de Saint Phalle, 2014, pp. 102-109, pp. 108-109.

\section{RÉSUMÉS}

Entre l'ouverture de l'École des beaux-arts aux memmes à la fin du XIX ${ }^{\mathrm{e}}$ siècle et l'attaque de la suffragette Mary Richardson contre la Toilette de Vénus de Vélázquez à l'aube de la Première Guerre mondiale, on assiste à une multiplication de discours mettant en scène la violence des femmes. La pratique d'un art physiquement engageant comme la sculpture et la révolte contre la répression des suffragettes donnent naissance à une iconographie évoquant celle, vétérotestamentaire, de Jaël et de Siséra, par la peintre Gentileschi. La représentation de la femme armée en vient à incarner la reconnaissance institutionnelle des femmes artistes, que ce 
soit de manière méliorative ou dépréciative, selon la tentative de contrer la "menace » symbolique que représentait cette reconnaissance nouvelle.

Between the opening of the École des beaux-arts to women in the late $19^{\text {th }}$ century and the attack by suffragette Mary Richardson at the dawn of World War I against Velázquez's The Toilet of Venus, commonly known in English as the Rokeby Venus, there was an abundance of speeches on the subject of the violence of women. The practice of a physically demanding art such as sculpture and the revolt against the repression of the Suffragettes stimulated an iconography that made reference to Gentileschi's depiction of the Old Testament story of Jael and Sisera. The representation of armed women came to symbolise the institutional recognition of women artists, either in a meliorative or depreciating way, depending on the attempt made to counter the symbolic "threat" that this this new recognition posed.

\section{INDEX}

Keywords : Camille Claudel, Artemisia Gentileschi, sculpture, gender studies, Suffragettes, Jael, Hélène Bertaux, sculptresses, iconology

Mots-clés : Camille Claudel, Artemisia Gentileschi, Sculpture, études de genre, Suffragettes, Jaël, Hélène Bertaux, sculptrices, iconologie

\section{AUTEUR}

\section{EVA BELGHERB}

Depuis 2017, Eva Belgherbi est doctorante en histoire de l'art à l'Université de Poitiers et à l'École du Louvre, elle est dirigée par Claire Barbillon et Amélie Simier et son sujet de thèse porte sur l'enseignement de la sculpture aux femmes, en France et au Royaume-Uni (1863-1914). Elle a coorganisé les journées d'étude « Violent(e)s, genre et violence dans l'histoire de l'art » à l'École du Louvre et communique régulièrement sur son travail lors de colloques internationaux. Elle collabore avec l'association AWARE (Archives of Women Artists, Research and Exhibitions). Since 2017 Eva Belgherbi has been a doctoral student in the history of art at the Université de Poitiers and the École du Louvre, under the direction of Claire Barbillon and Amélie Simier, and her dissertation analyses the teaching of sculpture to women in France and the United Kingdom (1863-1914). She was a co-organiser of the "Violent(e)s, genre et violence dans l'histoire de l'art" study days at the École du Louvre and regularly speaks about her work at international symposia. She contributes to the association AWARE (Archives of Women Artists, Research and Exhibitions). 